\title{
Conformal Bacterial Cellulose Coatings as Lubricious Surfaces
}

Patrick A. Rühs ${ }^{1,2}$, Katerina G. Malollari' ${ }^{3}$, Marco R. Binelli1, Rowena Crockett ${ }^{4}$, Diederik W. R. Balkenende ${ }^{2}$, André R. Studart ${ }^{*}$, and Phillip B. Messersmith ${ }^{2,5,6 *}$

${ }^{1}$ Complex Materials, Department of Materials, ETH-Zurich/Swiss Federal Institute of Technology, 8093, Zurich, Switzerland.

2 Department of Bioengineering, University of California, Berkeley, Berkeley 947201760, CA, USA.

${ }^{3}$ Department of Mechanical Engineering, University of California, Berkeley, Berkeley 94720-1760, CA, USA.

${ }^{4}$ Nanoscale Materials Science, Swiss Federal Laboratories for Materials Science and Technology, Zurich 8600, Switzerland

${ }^{5}$ Department of Materials Science and Engineering, University of California, Berkeley, Berkeley 94720-1760, CA, USA.

${ }^{6}$ Materials Sciences Division, Lawrence Berkeley National Laboratory, Berkeley 94720-1760, USA.

*Co-corresponding authors: philm@berkeley.edu; andre.studart@mat.ethz.ch;

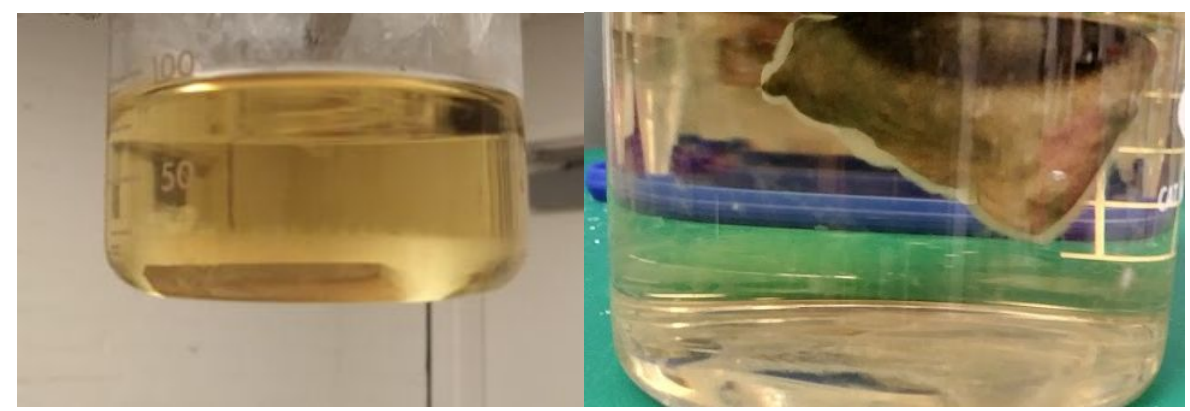

Supporting Figure S1: cBC coatings around 3D objects. Nylon substrates with attached bacteria were incubated in Mannitol-based media for 24 hours. The fuzzy layer around the flat (left) and 3D substrate (right) is clearly visible around the 3D object.

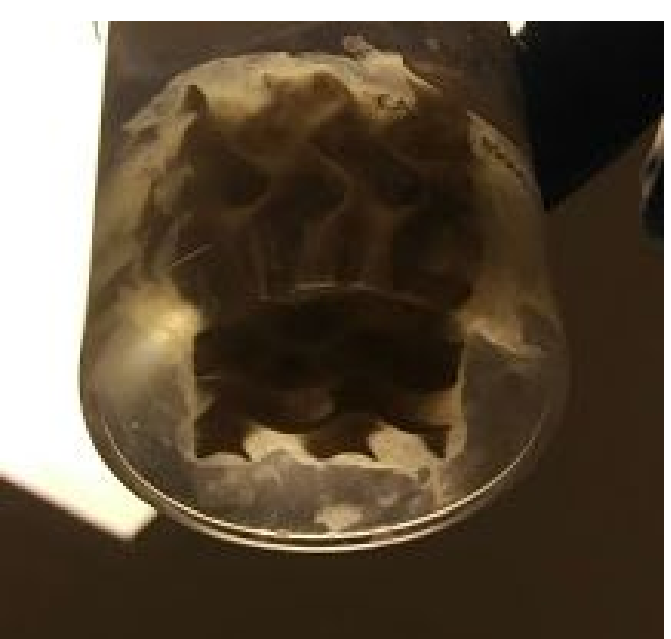


Supporting Figure S2: 3D substrate coated with bacterial cellulose (bottom view). Bacterial cellulose growth is not limited by oxygen and therefore occurs throughout the whole material.
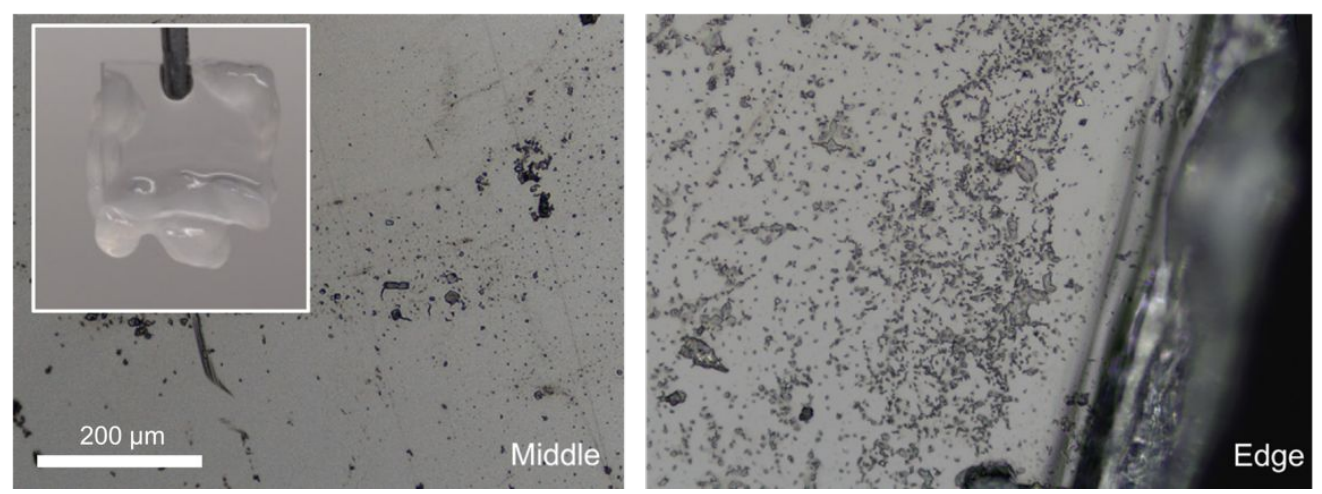

Supporting Figure S3: Microscopy images of polycarbonate (PC) with adhered bacteria on the surface. PC (inset) surface with adhered bacteria before growth in the middle and near the edge of a polycarbonate) PC piece. (inset) Polycarbonate piece after bacterial cellulose formation. 


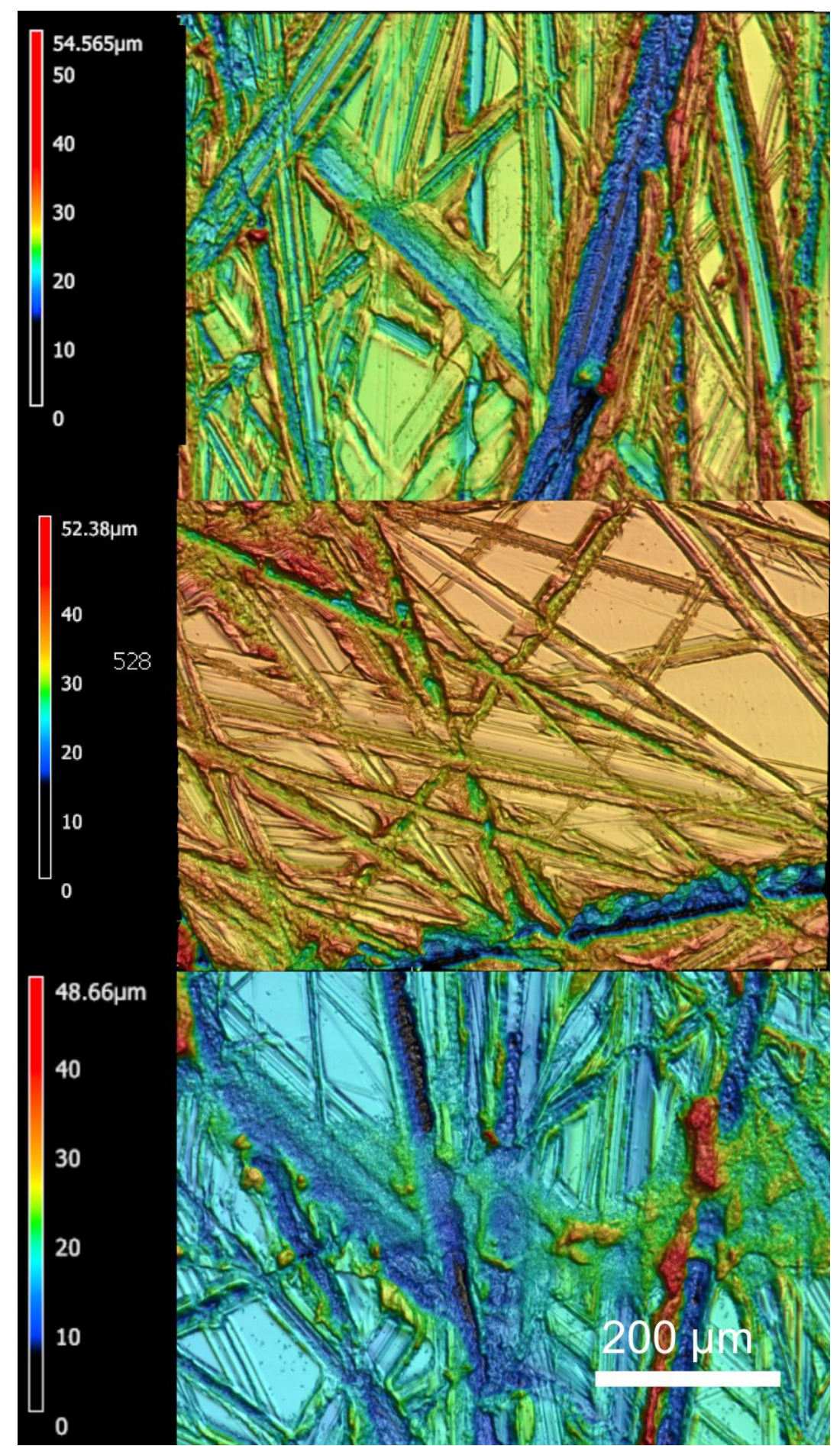

Supporting Figure S4: 3D profiles of rough nylon with attached bacteria before incubation in media. 


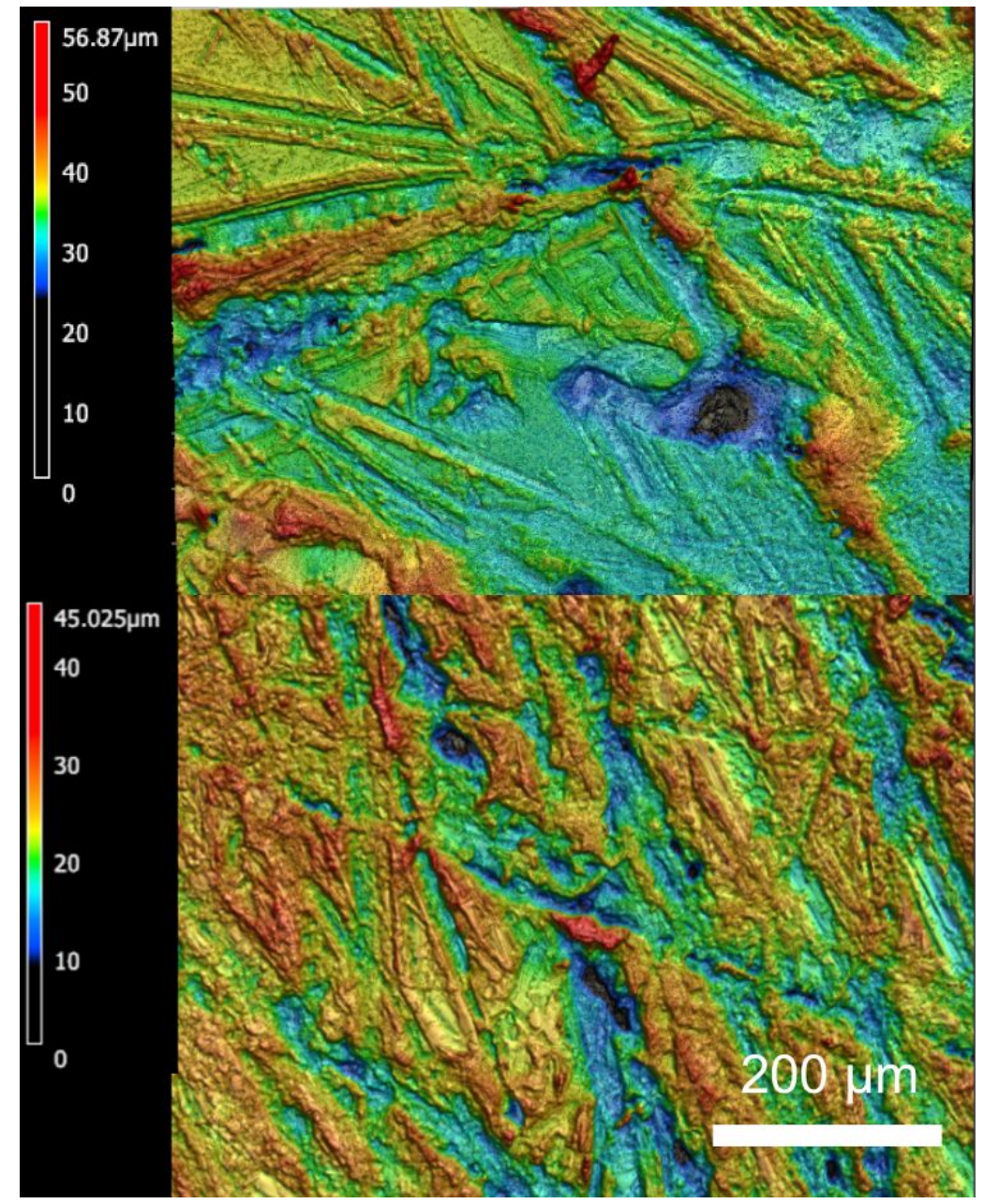

Supporting Figure S5: 3D profiles of rough nylon with attached bacteria before incubation in media. The rough nylon was pre-coated with polydopamine.

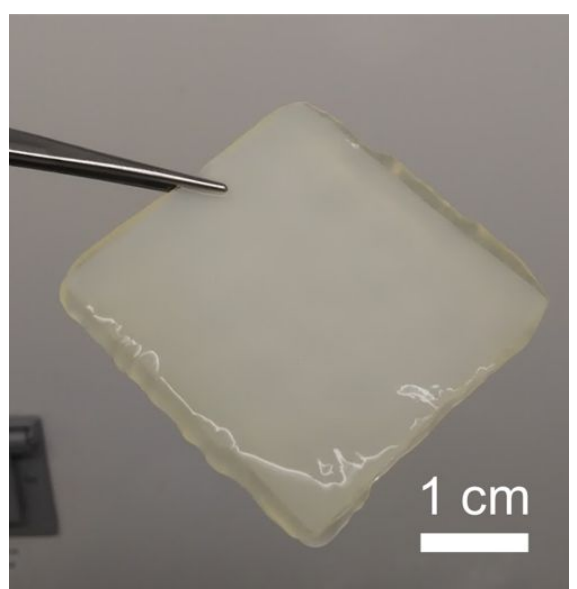

Supporting Figure S6: Bacterial cellulose coating (flat) cultured in non-shaking conditions to form flat and thin conformal bacterial cellulose coatings. 


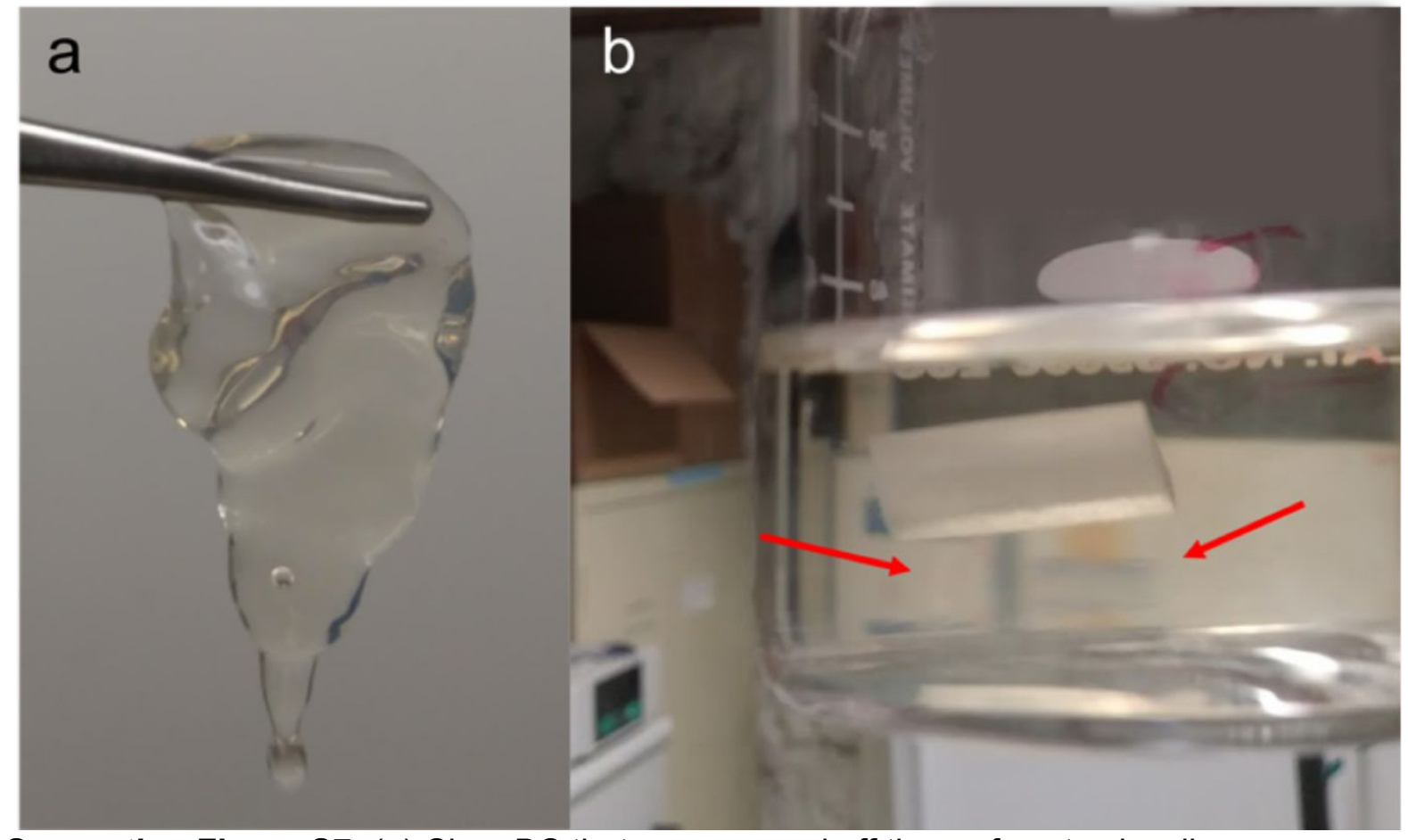

Supporting Figure S7: (a) Clear BC that was scraped off the surface to visualize transparency and (b) before scraping off the object. Here the object is suspended on the gel to demonstrate the transparency of the BC.
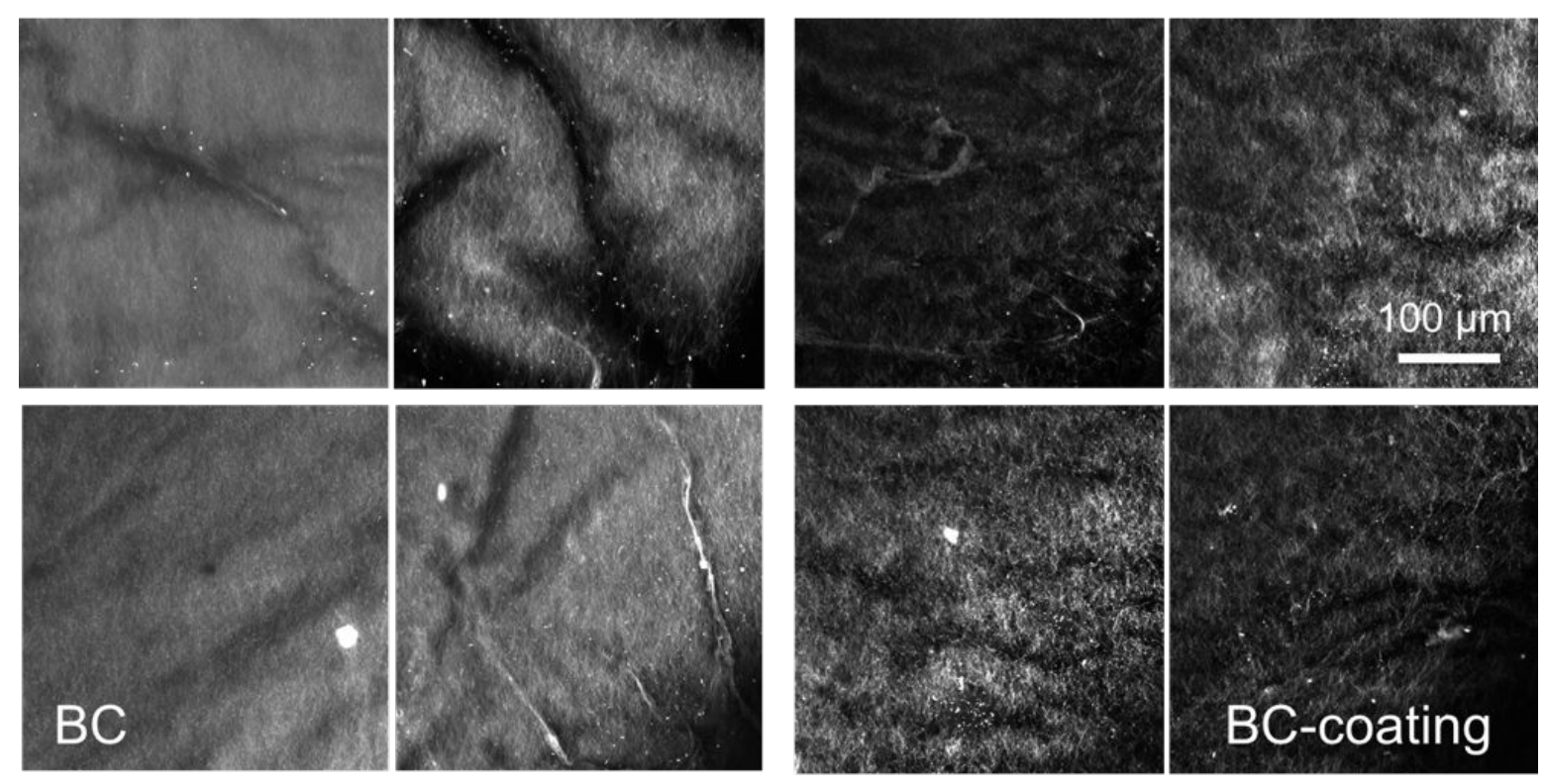

Supporting Figure S8: Confocal Laser Scanning Microscopy (CLSM) of (BC) films and cBC coatings. 


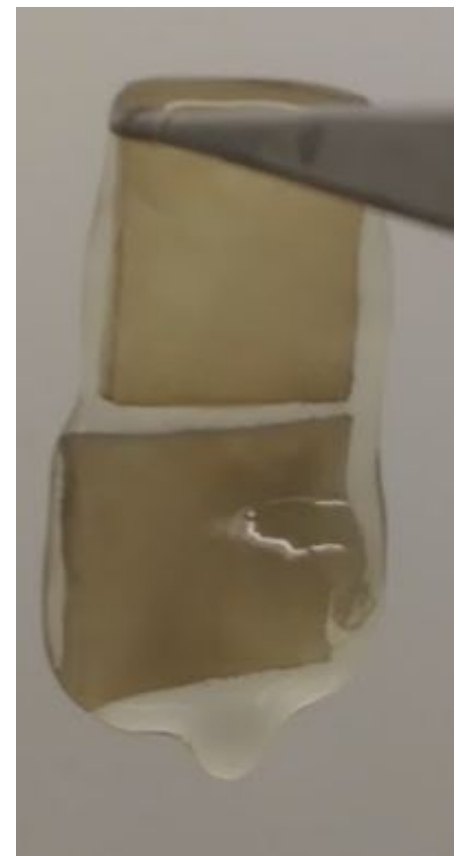

Supporting Figure S9: Bio-welded nylon struts with BC grown in close proximity to each other.

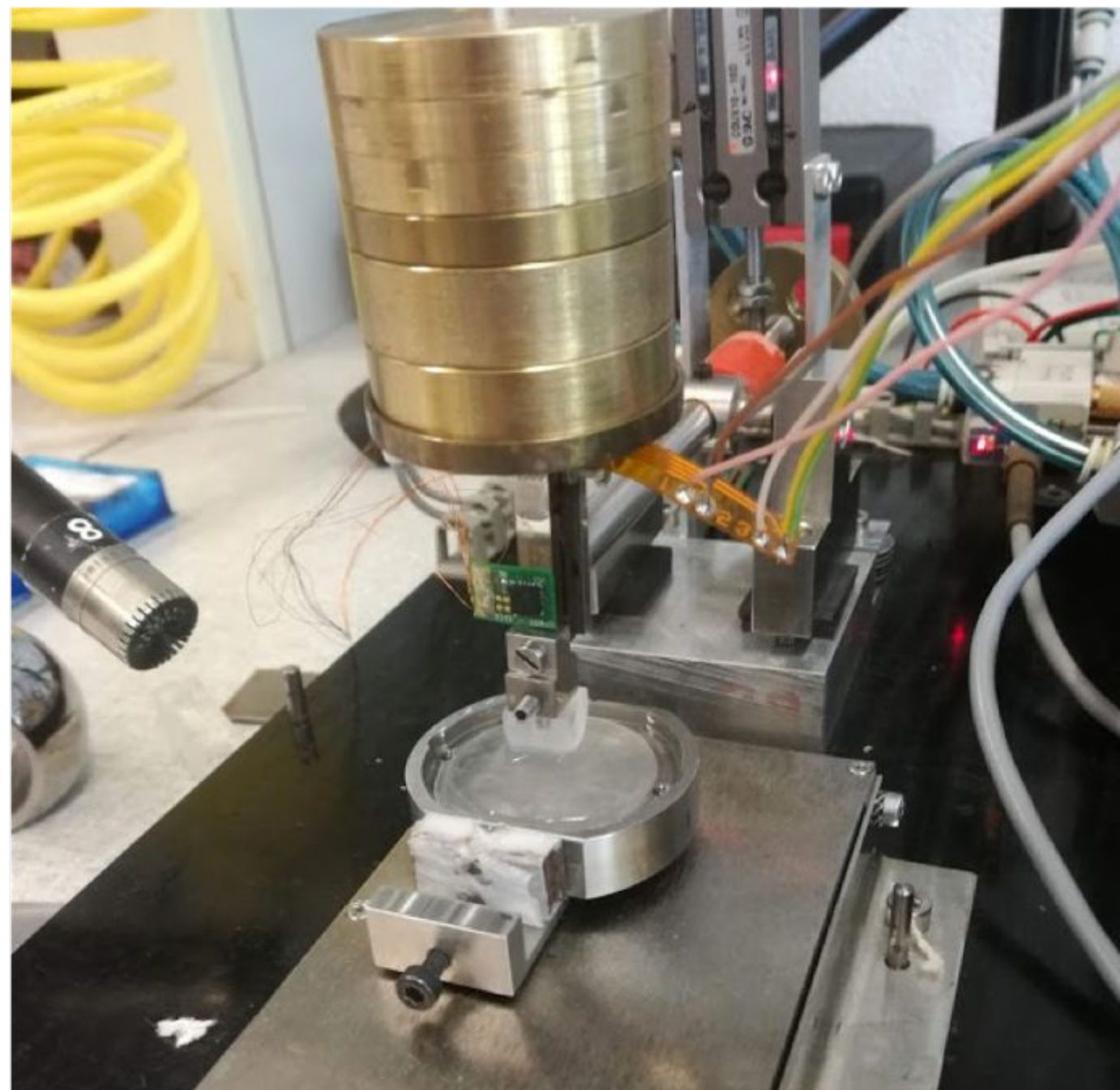

Supporting Figure S10: Setup of the tribological experiments done on the wet-vs-wet cBC coatings. 


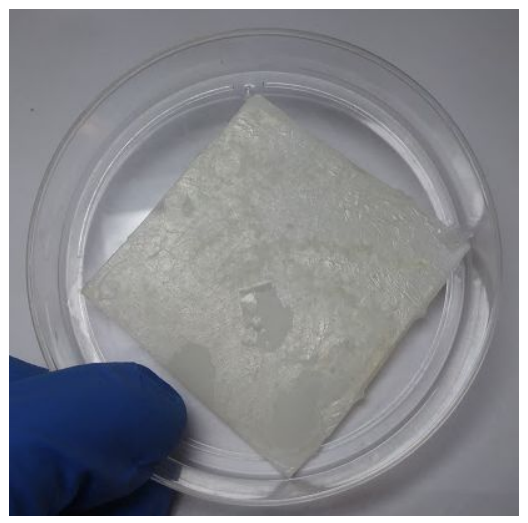

Supporting Figure S11: Dried down BC film on rough nylon. To demonstrate the thickness of the coating, the middle part is scratched free of bacterial cellulose.
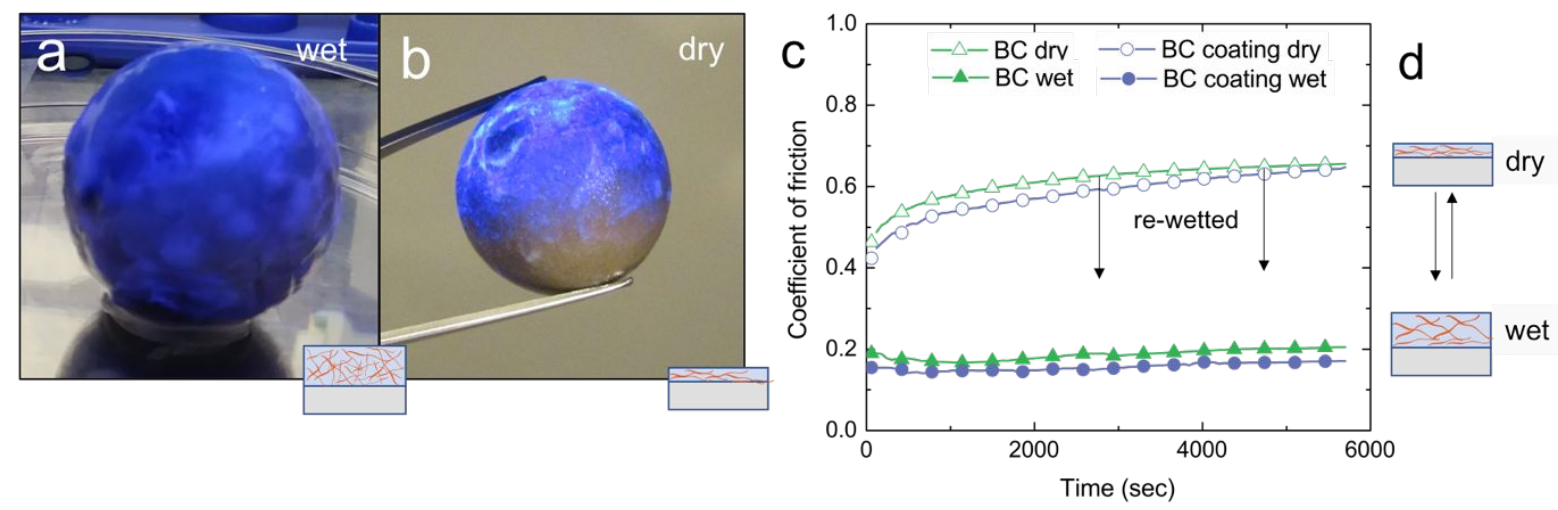

Supporting Figure S12: BC coated nylon sphere wet (a) and dry (b). (c) coefficient of friction of $\mathrm{BC}$ films and $\mathrm{CBC}$ coatings in the dry and re-hydrated state. (d) Schematic of the coating thickness during drying and re-wetting.

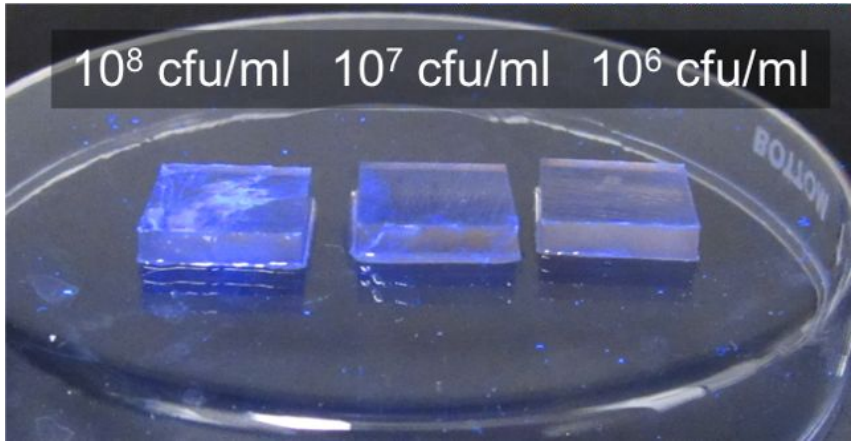

Supporting Figure S13: Initial bacteria concentration $\left(10^{8}-10^{6} \mathrm{cfu} / \mathrm{ml}\right)$ and its effect on bacterial cellulose formation on polydopamine rough polycarbonate (PC).

\section{Supporting Information S1:}

$\mathrm{BC}$ coatings form slippery-when-wet layers on 3D objects. To determine the coefficient of friction of $\mathrm{BC}$ and $\mathrm{BC}$ coatings, we used a tribometer with sliding pairs operating a $200 \mathrm{mN}$ normal load. Supporting Figure S12c shows the representative coefficient of friction (COF) of the dry and rehydrated BC and BC coatings. The COF for the dry samples is characterized by two friction values; initial stages show lower 
COF values and as sliding continues the COF gradually increases. This behavior can be possibly attributed to the wear occurring in the dried films as drying induces roughness. BC and BC dried coatings have an identical coefficient of friction of 0.6. In the dried state, both bacterial cellulose networks look identical, forming a thick mesh of bacterial cellulose fibrils. When the hydrophilic BC fibers are rewetted, they soak up water to a certain extent and form a slippery-when-wet layer with a coefficient of friction $(0.2)$ three times lower than in the dry state. Such frictional response can be attributed to the amount of liquid (lubricant) that the compliant gel can expel during the contact pressure. ${ }^{1}$ Both films demonstrated a steady state friction regime for long test duration, which indicates that both surfaces remain relatively preserved even after 2 hours under reciprocating loading. The coefficient of friction is highly dependent on the choice of material, friction partners, and on the BC drying history. Lopez et al., ${ }^{2}$ have for example demonstrated that friction similar to cartilage $(0.05)$ can be achieved when measuring never-dried $\mathrm{BC}$ against cartilage in the wet state at $37^{\circ} \mathrm{C}$ with a minimal degree of abrasion. Reinforced $\mathrm{BC}$ hydrogels with gelatin have also shown that coefficient of frictions as low as 0.001 are possible when measuring against glass. ${ }^{3}$

\section{References:}

(1) Adams, M. J.; Briscoe, B. J.; Johnson, S. A. Friction and Lubrication of Human Skin. Tribol. Lett. 2007, 26, 239-253.

(2) Lopes, J. L.; Machado, J. M.; Castanheira, L.; Granja, P. L.; Gama, F. M.; Dourado, F.; Gomes, J. R. Friction and Wear Behaviour of Bacterial Cellulose against Articular Cartilage. Wear 2011, 271, 2328-2333.

(3) Nakayama, A.; Kakugo, A.; Gong, J. P.; Osada, Y.; Takai, M.; Erata, T.; Kawano, S. High Mechanical Strength Double-Network Hydrogel with Bacterial Cellulose. Adv. Funct. Mater. 2004, 14, 1124-1128. 